J. Lake Sci. (湖泊科学), 2020, 32(4): 1050-1059

DOI 10. 18307/2020. 0414

(c) 2020 by Journal of Lake Sciences

\title{
太湖蓝藻碎屑对水质及附着藻和水丝蚓生物量的影响”
}

\author{
夏曼莉 ${ }^{1,2}$, 于谨磊 $^{2 * *}$, 何 虎 $^{2}$,关保华 ${ }^{2,3}$, 陈非洲 ${ }^{2,3}$, 刘正文 ${ }^{1,2,3 * *}$ \\ (1:暨南大学生态学系水生生物研究所,广州 510632) \\ (2: 中国科学院南京地理与湖泊研究所湖泊与环境国家重点实验室,南京 210008) \\ (3: 中国科学院大学中丹科研教育中心, 北京 100049)
}

\begin{abstract}
摘 要: 富营养化是现今各国面临的主要水环境问题, 其中蓝藻水华暴发是全球富营养化水体最常见的现象之一. 蓝藻 水华将产生大量的蓝藻碎屑, 其对水质及生物的影响还尚不清楚. 本研究通过向中宇宙系统添加微囊藻碎屑, 分析其对 水体不同形态营养盐及水生生物生物量的影响. 结果表明: 微囊藻碎屑加人后, 水体不同形态的营养盐浓度均在短期内 迅速增加,其中水体总氮和总磷平均浓度最高分别达到 3.86 和 $0.36 \mathrm{mg} / \mathrm{L}$; 浮游植物生物量 (用叶绿素 $a$ 表示) 在前 9 天 随营养盐浓度的升高而增加, 随后逐渐下降至实验初始水平. 此外, 附着藻类生物量在微囊藻碎屑加人后呈逐渐下降趋 势, 这可能与浮游植物快速增殖引起的水体透明度下降有关. 微囊藻碎屑加人后, 水丝蚛生物量随微囊藻碎屑的分解持 续增长, 在第 20 天达到生物量最大值. 本研究通过模拟太湖梅梁湾生态系统, 探讨微囊藻碎屑对水质及水生生物生物量 的影响,结果有助于进一步了解蓝藻水华对水生态系统影响的途径及机理,为富营养化湖泊管理提供理论依据.
\end{abstract}

关键词: 太湖;蓝藻水华;营养盐释放;浮游植物;附着藻;水丝蚓

\section{Effects of cyanobacterial detritus on water quality and the biomass of periphyton and Lim- nodrilus spp. in Lake Taihu*}

\author{
XIA Manli ${ }^{1,2}$, YU Jinlei ${ }^{2 * *}$, HE Hu$^{2}$, GUAN Baohua ${ }^{2,3}$, CHEN Feizhou ${ }^{2,3}$ \& LIU Zhengwen ${ }^{1,2,3 * *}$ \\ (1: Department of Ecology and Institute of Hydrobiology, Jinan University, Guangzhou 510632, P.R. China) \\ (2: State Key Laboratory of Lake Science and Environment, Nanjing Institute of Geography and Limnology, Chinese Academy \\ of Sciences, Nanjing 210008, P.R. China) \\ (3: Sino-Danish Center for Education and Research, University of Chinese Academy of Sciences, Beijing 100049, P.R.China)
}

\begin{abstract}
Eutrophication has become a major water environmental problem all over the world, and cyanobacterial blooms are one of the most common phenomena in eutrophic lakes. Thereafter, a large amount of cyanobacterial detritus will be produced after the blooms, and the effects of these detritus on water quality and biological communities are poorly studied. A 28-day mesocosm experiment was conducted in Meiliang Bay of Lake Taihu to study the effects of cyanobacterial detritus on nutrients dynamics and the biomass of aquatic organisms. We found that the concentration of nutrients increased rapidly after addition of Microcystis detritus, with the maximum mean concentration of total nitrogen ( TN ) and total phosphorus ( TP) up to $3.86 \mathrm{mg} / \mathrm{L}$ and $0.36 \mathrm{mg} / \mathrm{L}$, respectively. Meanwhile, phytoplankton biomass (Chl.a) increased dramatically, after detritus addition, in the first nine days, then it gradually declined to the end of the experiment. In addition, the biomass of periphyton decreased gradually after cyanobacterial detritus addition which may be mainly due to the decreasing water transparency caused by the rapid increase of Chl. $a$ limiting the growth of periphyton. Meanwhile, the biomass of Limnodrilus spp. increased gradually, and peaked on day 20, indicating that cyanobacterial detritus facilitates the population development of Limnodrilus spp. Our results have implications for eutrophic lake management and better understanding the effects of cyanobacterial blooms on aquatic ecosystems.
\end{abstract}

Keywords: Lake Taihu; cyanobacterial bloom; nutrient release; phytoplankton; periphyton; Tubificidae

* 2019-11-01 收稿; 2020-01-01 收修改稿.

国家水体污染控制与治理科技重大专项 (2017ZX07203-004) 和国家自然科学基金项目 (41877415)联合资助.

** 通信作者; E-mail: jlyu@ niglas.ac.cn, zliu@ niglas.ac.cn. 
富营养化是现今各国面临的主要水环境问题, 其中蓝藻水华暴发是全球富营养化水体最常见的现象之 一. 外源污染物输人和内源营养盐释放将直接导致水体营养盐浓度升高, 促进浮游植物的生长繁殖. 由于蓝 藻自身的生理特征, 如具有伪空胞 (gas vesicle)、能够抵抗高光强和紫外线的干扰 等 $^{[-3]}$, 常在富营养化水体 中发展为浮游植物的优势类群. 蓝藻水华形成后, 有害藻类能够释放藻毒素, 影响水生生物和人类的健 康 ${ }^{[4-5]}$. 蓝藻水华消亡后, 将形成大量的蓝藻碎屑, 其腐烂分解后将向水体释放氮、磷等营养盐 ${ }^{[6-8]}$, 增加水体 的氮、磷负荷, 刺激浮游植物生长. 此外, 蓝藻碎屑还可作为水生生物的食物来源 ${ }^{[9-10]}$, 其对水生生物生物量 的影响还尚不清楚. 因此,蓝藻碎屑可从多个方面影响湖泊生态系统的结构与功能.

有关蓝藻碎屑对水域生态系统的影响研究,多关注其对水体水质的影响 ${ }^{[1-12]}$, 近年来也有学者通过野 外调查或历史数据分析等手段探讨蓝藻水华对水生生物的影响 ${ }^{[13-14]}$. 而通过模拟试验探究蓝藻碎屑对水生 生物影响的研究相对较少, 大多针对单一物种进行 ${ }^{[6,15-18]}$. 本试验从探究蓝藻水华在水体中的影响出发, 通 过向中宇宙受控系统添加微囊藻碎屑, 分析其对水体营养盐和水域生态系统中不同的生物类群生物量 (浮 游植物、附着藻类、水丝蚓) 的影响. 研究结果将为进一步研究蓝藻水华对水生生态系统的影响提供参考, 同 时为富营养化湖泊管理提供理论依据.

\section{1 材料与方法}

\section{1 试验设置}

微囊藻碎屑制备: 在夏季 ( 8 月), 用孔径为 $64 \mu \mathrm{m}$ 的浮游生物网在太湖梅梁湾采集足量的新鲜微囊藻 (实验室镜检发现以铜绿微囊藻为主,占总体积的 $99 \%$ ) : 在实验室, 用蒸馏水洗涤采集的新鲜微囊澡并去 除藻液中的杂质, 随后将微囊藻置于 $60^{\circ} \mathrm{C}$ 烘箱烘至恒重. 最后, 用研针将烘干的微囊藻碎屑研磨成粉, 经 $300 \mu \mathrm{m}$ 耖网过滤后, 置于干燥器内备用. 此外, 在微囊藻放人烘箱前, 我们取样测定了微囊藻单位湿重生物 量的叶绿素含量, 结合微囊藻藻浆含水率, 计算得出 $1 \mathrm{~g}(\mathrm{DW})$ 微囊藻碎屑相当于 $50 \mu \mathrm{g} / \mathrm{L}$ 的新鲜微囊藻叶 绿素 $a$ 浓度.

中宇宙试验系统构建: 本试验在位于梅梁湾的湖泊生态系统实验站进行. 为研究微囊藻碎屑对水质及 水生生物生物量的影响随时间的变化过程, 本试验共设置 24 个由高密度聚乙烯塑料桶组成的中宇宙试验 系统 (容积: $130 \mathrm{~L}$; 高: $66 \mathrm{~cm}$; 上口直径: $56 \mathrm{~cm}$; 底部直径: $45 \mathrm{~cm}$ ) : 每个试验系统内加人 $15 \mathrm{~cm}$ 混匀的太 湖梅梁湾沉积物 (采自离岸 $200 \mathrm{~m}$ 的表层 $20 \mathrm{~cm}$ 沉积物, 经孔径为 $0.5 \mathrm{~cm}$ 的篮网过滤) 和 $90 \mathrm{~L}$ 经 $64 \mu \mathrm{m}$ 浮 游生物网过滤的湖水. 试验系统置于梅梁湾岸边的水泥池中 (规格: $6 \mathrm{~m} \times 5 \mathrm{~m} \times 2 \mathrm{~m}$ ), 固定试验桶并使其漂浮 于池塘水面(图 1).

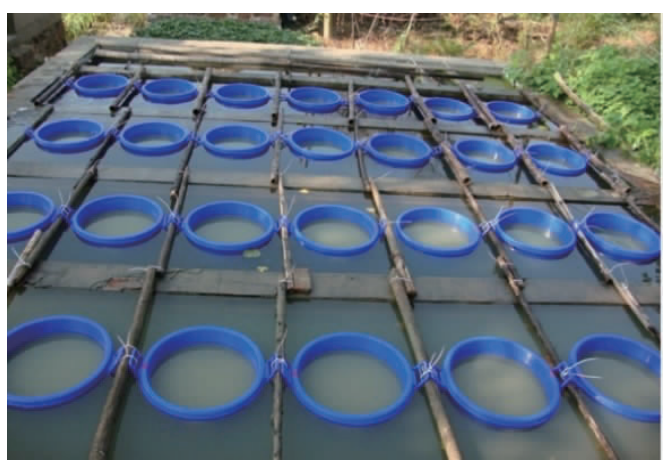

图 1 中宇宙试验系统设置

Fig.1 Mesocosm experiment setup

为使试验系统尽可能地接近梅梁湾生态系统, 试验开始前, 参考太湖现存底栖动物生物量 ${ }^{[19-21]}$, 向每个 中宇宙试验系统加人了河蚬 Corbicula fluminea (总数量为 $3 \sim 4$ 个, 平均总重量为 $12 \mathrm{~g}$ )、铜锈环棱螺 Bellamya aeruginosa (总数量为 $6 \sim 7$ 个, 平均总重量为 $10 \mathrm{~g}$ )、椭圆萝卜螺 Radix swinhoei (总数量为 4 个, 平均总重量为 
$1.1 \mathrm{~g}$ )、扭蚌 Arconaia lanceolata (总数量为 $1 \sim 2$ 个, 平均总重量为 $18.6 \mathrm{~g}$ ) 、背角无齿蚌 Anodonta woodiana (总 数量为 1 个, 平均总重量为 $26.9 \mathrm{~g}$ ) 和圆顶珠蚌 Unio douglasiae (总数量为 $1 \sim 2$ 个, 平均总重量为 $26.8 \mathrm{~g}$ ), 而 水丝蚂则由加人系统中的沉积物娐化而来.

本试验在 8-9 月份进行, 试验期间平均水温为 $30^{\circ} \mathrm{C}$, 该试验共持续 28 天. 试验开始前, 系统经过 1 个 月的稳定沉降, 试验桶内水体水质指标相对稳定且无显著差异; 总氮 ( TN ) 浓度为 $1.17 \pm 0.37 \mathrm{mg} / \mathrm{L}$ 、总磷 (TP) 浓度为 $0.051 \pm 0.012 \mathrm{mg} / \mathrm{L} 、$ 叶绿素 $a($ Chl. $a)$ 浓度为 $25.37 \pm 4.15 \mu \mathrm{g} / \mathrm{L}$. 试验开始时, 向 21 个试验系统中 添加 $7 \mathrm{~g}$ 微囊藻碎屑 (相当于新鲜微囊藻 Chl. $a$ 浓度为 $350 \mu \mathrm{g} / \mathrm{L}$ ), 其模拟的蓝藻水华 Chl. $a$ 浓度在蓝藻水华 大面积暴发时的 Chl. $a$ 浓度范围之间 (78.3 978.5 $\mu \mathrm{g} / \mathrm{L})^{[22]}$; 剩余 3 个试验系统不添加微囊藻碎屑, 作为本 试验的初始值 (对照组).

\section{2 样品采集与分析}

在试验开始的第 $0 、 1 、 3 、 5 、 9 、 14 、 20$ 和 28 天进行采样. 第 0 天采集的样品作为试验本底值,随后每次采 样, 随机采集剩余试验系统中 3 个桶中的上覆水样、底栖动物和附着藻类. 为了更精确地计算筒壁附着藻类 生物量和水丝蚓生物量, 每次采样均采取破坏性采样的方式, 即采样结束后, 试验桶将被清空.

采用虹吸法收集 $5 \mathrm{~L}$ 不同水层的上覆水,带回实验室分析水体不同氮、磷形态的营养盐浓度和 Chl. $a$ 浓 度. 本试验中 $\mathrm{TN}$ 、溶解性总氮 $(\mathrm{TDN})$ 、氨氮 $\left(\mathrm{NH}_{3}-\mathrm{N}\right)$ 、硝态氮 $\left(\mathrm{NO}_{3}^{-}-\mathrm{N}\right) 、 \mathrm{TP}$ 、溶解性总磷 $(\mathrm{TDP})$ 和溶解性反应 磷 (SRP) 的分析方法参照《湖泊富营养化调查规范》 ${ }^{[23]}$. 其中, TN 、TDN、TP、TDP 浓度采用碱性过硫酸钾联 合消解法测定; $\mathrm{NH}_{3}-\mathrm{N}$ 浓度采用纳氏试剂比色法测定; $\mathrm{NO}_{3}^{-}-\mathrm{N}$ 浓度采用紫外分光光度法测定; SRP 浓度采 用钿一锑一抗分光光度法测定. 用醋酸纤维膜过滤 $0.5 \sim 1 \mathrm{~L}$ 水样, 将滤膜转入离心管, 加人 $10 \mathrm{~mL} 90 \%$ 的丙酮 溶液, 充分震荡后置于 $4^{\circ} \mathrm{C}$ 暗处反应 $18 \sim 24 \mathrm{~h}$; 随后, 经 $3500 \mathrm{r} / \mathrm{min}$ 离心后, 取上清液, 用紫外分光光度计测 定其在 750、663、645 和 $630 \mathrm{~nm}$ 波长下的吸光值,计算水样中的 Chl. $a$ 浓度.

水丝蚛和附着藻类样品在试验系统中的水被虹吸完毕后进行. 水丝蚓样品采集时, 用 60 目尼龙篮网过 滤系统内的所有沉积物, 在实验室内挑取水丝蚂, 用蒸馏水冲洗后置于 $60^{\circ} \mathrm{C}$ 烘箱中烘至恒重, 计算其生物 量. 试验系统内壁表面附着藻类采集时, 将桶壁附着藻用刷子刷入烧杯中, 在实验室用 GF/C 膜抽滤混匀后 的附着藻液,然后将膜置于 $60^{\circ} \mathrm{C}$ 烘箱中烘至恒重,称量并计算其生物量.

\section{3 数据分析}

本研究的所有数据统计分析均在 SPSS 20.0 软件中进行. 采用 $t$ 检验比较微囊藻碎屑加人后, 每次采样 中不同形态营养盐、Chl. $a$ 、附着藻类生物量和水丝蚂生物量与对照组 (第 0 天的数据) 间的差异, 从而分析各 采样时间点样品与初始样品的差异.

\section{2 结果}

\section{1 微囊藻碎屑对水体营养盐的影响}

2.1.1 水体不同形态氮浓度的变化过程 微囊藻碎屑加人后, 水体的 TN 浓度变化明显. TN 浓度在碎屑加人 后第 1 天即迅速上升, 平均浓度从初始的 $1.0 \mathrm{mg} / \mathrm{L}$ 上升至 $3.5 \mathrm{mg} / \mathrm{L}$ (图 $2 \mathrm{~A}$ ). 随后, $\mathrm{TN}$ 浓度略有下降, 但第 3 天 $(t=4.30, P=0.012)$ 和第 5 天 $(t=4.30, P=0.009)$ 的浓度仍显著高于初始浓度 (图 $2 \mathrm{~A})$. 随后, $\mathrm{TN}$ 浓度继 续上升, 直至第 14 天时达到最高值, 并在剩余的试验期内维持在较高的浓度水平 (3.3 3.9 mg/L) (图 $2 \mathrm{~A}$ ).

微囊藻碎屑加人引起的水体 TN 浓度升高, 主要以溶解态氮的形式存在. TDN 浓度在微囊藻碎屑加人后 呈明显上升趋势 (图 2B) , 第 3 天的浓度达到 $3.0 \mathrm{mg} / \mathrm{L}$. 第 5 和第 9 天的 TDN 浓度略低于第 3 天的浓度,随 后 TDN 的浓度在试验结束前一直呈上升趋势 (图 2B). 试验期间, TDN 浓度始终显著高于初始 TDN 浓度 $(P<0.05)$. 本试验分析了 TDN 的两种形态氮浓度 (分别是 $\mathrm{NH}_{3}-\mathrm{N} 、 \mathrm{NO}_{3}^{-}-\mathrm{N}$ ), 其中以 $\mathrm{NH}_{3}-\mathrm{N}$ 的形态为主, 其浓 度在微囊藻碎屑加人后持续上升, 在第 3 天时平均浓度达到 $2.0 \mathrm{mg} / \mathrm{L}$. 随后, $\mathrm{NH}_{3}-\mathrm{N}$ 浓度在第 5 天时降至 $1.2 \mathrm{mg} / \mathrm{L}$, 直至第 20 天一直维持在相近的浓度水平. 试验结束时, $\mathrm{NH}_{3}-\mathrm{N}$ 浓度再次升高至试验第 3 天的浓度 (图 2C). $\mathrm{NO}_{3}^{-}-\mathrm{N}$ 浓度在试验开始时一直呈上升趋势 (1 20 天), 试验第 14 天时, $\mathrm{NO}_{3}^{-}-\mathrm{N}$ 浓度显著高于初始 值 $(t=2.78, P=0.004)$, 一直到第 20 天时开始下降, 试验结束时, $\mathrm{NO}_{3}^{-}-\mathrm{N}$ 浓度仍然显著高于初始浓度 $(t=$ $2.78, P=0.029$ ) (图 2D). 

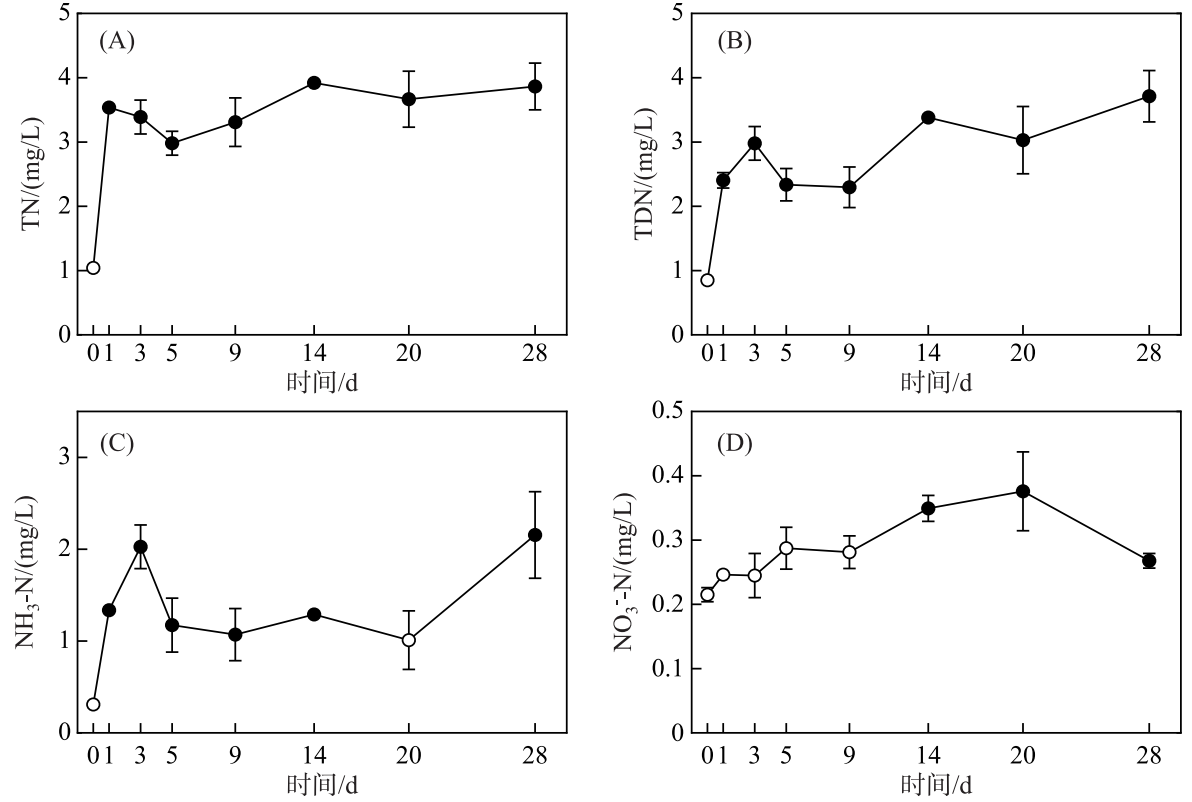

图 2 微囊藻碎屑加人后水体 $\mathrm{TN} 、 \mathrm{TDN} 、 \mathrm{NH}_{3}-\mathrm{N}$ 和 $\mathrm{NO}_{3}^{-}-\mathrm{N}$ 浓度的变化趋势

(图中实心符号表示该值与对照组 (第 0 天) 存在显著性差异)

Fig. 2 Changes of the concentrations of total nitrogen, total dissolved nitrogen, ammonia and nitrate in the water column of the mesocosms after addition of Microcystis detritus

( The filled symbols represent the concentration of a given nutrients form on days where it was significantly different from the control's values (on day 0))

2.1.2 水体不同形态磷浓度的变化过程 微囊藻碎屑加人后, 水体 TP 浓度的变化呈明显的双峰曲线 (图 $3 \mathrm{~A})$ : TP 浓度在碎屑加人后第 1 天平均浓度达到 $0.30 \mathrm{mg} / \mathrm{L}$, 然后 TP 浓度逐渐下降, 在试验第 5 天时降至 $0.19 \mathrm{mg} / \mathrm{L}$, 显著高于初始浓度 $(t=2.78, P=0.005)$. 随后, TP 浓度再次升高, 第 14 天达到最高值, 平均浓度 为 $0.36 \mathrm{mg} / \mathrm{L}$. 试验结束时 TP 平均浓度降至 $0.12 \mathrm{mg} / \mathrm{L}$ (图 3A). 试验前 20 天, TDP、SRP 与 TP 浓度的变化 趋势基本一致, 在试验前 3 天浓度显著升高, 试验第 5 天浓度降低至初始水平, 试验第 5 天后浓度继续上升, 呈现双峰曲线 (图 3B、C). 在试验结束时, 与对照组 (第 0 天) 相比, $\operatorname{TP}(t=2.78, P=0.023$ )、 $\operatorname{TDP}(t=2.78, P=$ 0.008 ) 仍显著高于初始值, SRP 浓度与初始值均无显著性差异 $(t=2.78, P=0.123)$ (图 3C).

\section{2 微囊藻碎屑对水生生物生物量的影响}

2.2.1 浮游植物生物量变化过程 试验开始阶段 (第 0 3 天), 浮游植物生物量 ( 叶绿素 $a$ 浓度) 未发生显著 变化 $(P>0.05)$ (图 4): 随着氮、磷营养盐浓度的快速升高, 水体 Chl. $a$ 浓度也迅速上升, 平均浓度在第 5 天 时达到 $50.4 \mu \mathrm{g} / \mathrm{L}$, 为初始值的 2 倍. 试验第 9 天时, Chl. $a$ 平均浓度达到试验期间的最高值, 为 $56.0 \mu \mathrm{g} / \mathrm{L}$. 随 后, Chl. $a$ 浓度开始逐渐下降, 平均浓度在试验第 14 天降至 $27.8 \mu \mathrm{g} / \mathrm{L}$, 在第 20 天出现小幅回升后继续下降. 到试验结束时, Chl. $a$ 浓度与初始值无显著性差异 $(t=2.776, P=0.51)$, 浓度为 $23.6 \mu \mathrm{g} / \mathrm{L}$ (图 4).

2.2.2 附着藻类生物量变化过程 微囊藻碎屑加人后的第 $1 、 3$ 和 5 天, 附着藻生物量与第 0 天无显著性差异 (图 5), 其平均生物量在试验第 3 天降至 $5.3 \mathrm{~g}(\mathrm{DW}) / \mathrm{m}^{2}$, 第 5 天重新升至 $7.9 \mathrm{~g}(\mathrm{DW}) / \mathrm{m}^{2}$. 随后, 附着藻生 物量一直呈下降趋势, 从试验开始的第 20 天开始直至结束, 附着藻生物量均显著低于第 0 天的生物量 $(P<$ 0.05) (图 5).

2.2.3 水丝蚂生物量变化过程 微囊藻碎屑加人后, 水丝蚂生物量在试验开始的前 14 天内变化相对稳定, 均 与第 0 天的生物量无显著性差异 $(P>0.05)$ (图 6). 而在第 20 天时, 水丝蚂生物量达到本试验的最高值, 显 著高于初始值 $(t=4.30, P=0.05)$, 平均为 $1.15 \mathrm{~g}(\mathrm{DW}) / \mathrm{m}^{2}$. 试验结束时, 水丝蚂生物量下降到 

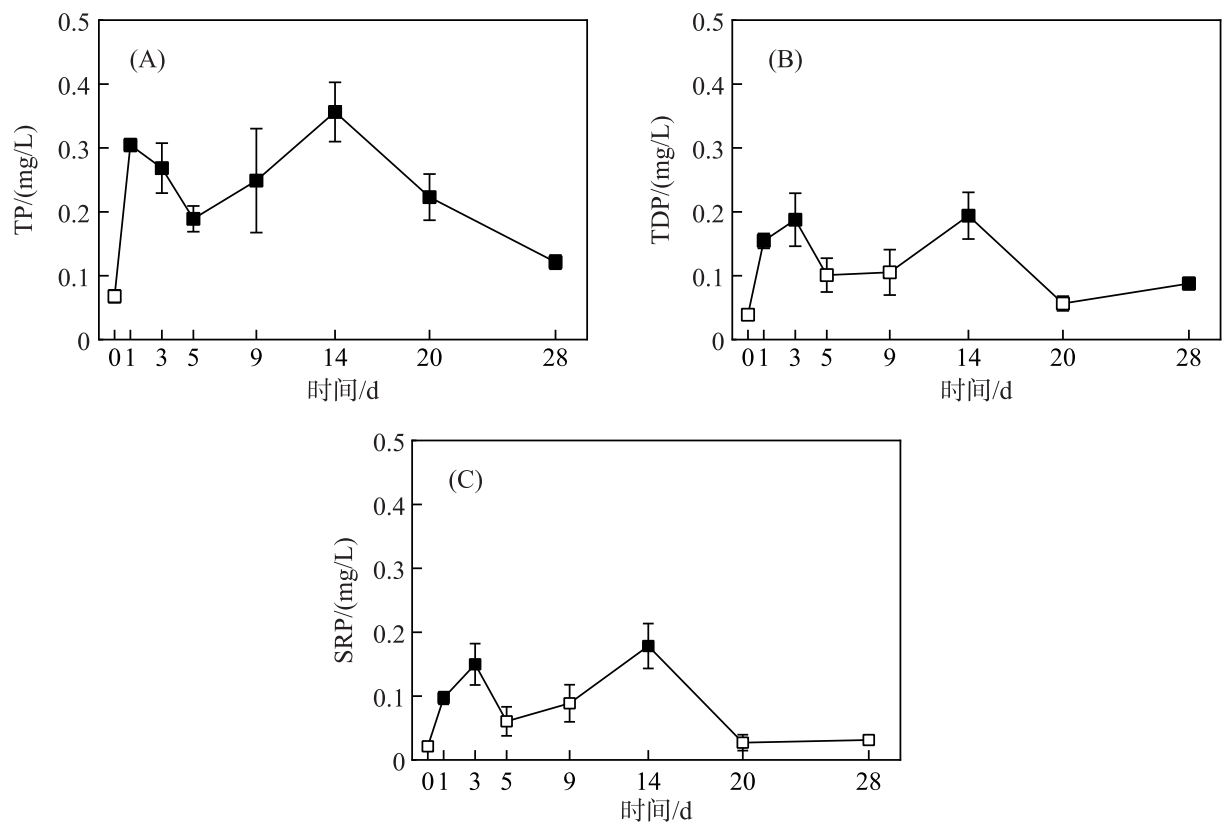

图 3 微囊藻碎屑加人后水体 TP、TDP 和 SRP 浓度的变化趋势 (图中实心符号表示该值与对照组 (第 0 天) 存在显著性差异)

Fig.3 Changes of the concentrations of total phosphorus, total dissolved phosphorus and soluble reactive phosphorus in the water column of the mesocosms after addition of Microcystis detritus

( The filled symbols represent the concentration of a given nutrients form on days where it was significantly different from the control's values (on day 0))

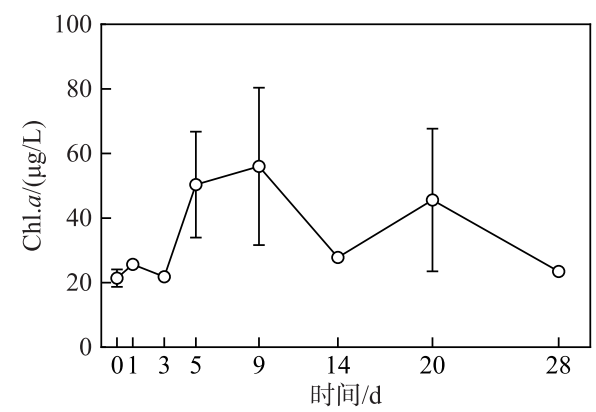

图 4 微囊藻碎屑加人后浮游植物生物量 (Chl. $a$ 浓度) 的变化趋势

Fig.4 Trends of the phytoplankton biomass (Chl.a concentration) after addition of Microcystis detritus

$0.37 \mathrm{~g}(\mathrm{DW}) / \mathrm{m}^{2}$, 显著高于试验开始 (对照组, 第 0 天) 时的生物量 $(t=2.78, P=0.029)$.

\section{3 讨论}

本研究通过中宇宙试验, 探讨微囊藻碎屑对水质及浮游植物、附着藻类和水丝蚓生物量的影响. 微囊藻 碎屑加人系统后, 水体营养盐在短时间内 (第 1 天) 显著上升; 浮游植物 (Chl. $a$ )、底栖动物和附着藻类生物 量也随之发生显著变化. 因此, 微囊藻碎屑在腐烂分解过程中, 不但影响水质, 而且还直接影响水生生物生 物量. 


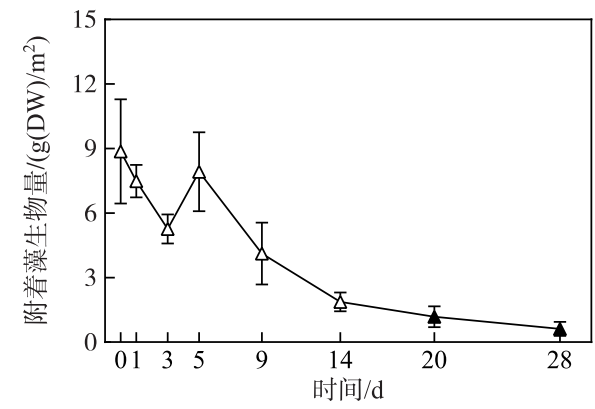

图 5 附着藻类生物量的变化趋势 (图中实心符号表示该值与对照组 (第 0 天)存在显著性差异)

Fig. 5 Changes of the biomass of periphyton after addition of Microcystis detritus (The filled symbols represent the periphyton biomass on days where it was significantly different from the control's values (on day 0)

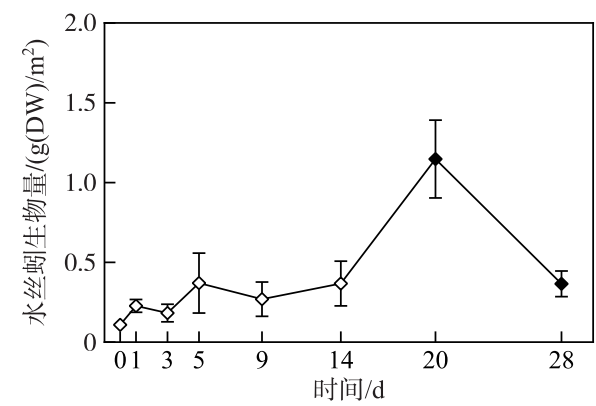

图 6 微囊藻碎屑加人后水丝蚓生物量的变化趋势 (图中实心符号表示该值与对照组 (第 0 天) 存在显著性差异)

Fig. 6 Changing pattern of the biomass of Tubificidae after addition of Microcystis detritus(The filled symbols represent the biomass on days where it was significantly different from the control's values (on day 0))

已有研究多通过野外调查或历史数据分析的方式研究微囊藻水华对水生生物群落的影响 ${ }^{[13-16]}$, 即在太 湖或巢湖等富营养化较为严重的湖区, 微囊藻水华暴发频繁, 耐污种 (例如水丝蚓) 的生物量高于富营养化 较轻的湖区. 本研究结果直接证实了微囊藻碎屑对浮游植物生物量和水丝蚓生物量增加的促进作用, 这与 已有的研究结果一致 ${ }^{[9,24]}$. 水丝蚓是淡水生态系统中常见的底栖动物, 生活在底泥表层, 以有机颗粒为食. 龚志军等 ${ }^{[25]}$ 、李艳等 ${ }^{[26]}$ 的野外调查发现: 水丝蚓密度随湖泊富营养化程度增加而上升, 水丝蚓密度的增加 与水体营养盐和沉积物有机质含量有关. 而微囊藻碎屑在分解过程中, 不仅增加了水体营养盐浓度, 而且分 解较慢或全分解的残渣, 会以有机颗粒的形式沉降到底泥表面, 增加了沉积物表面有机质含量, 为水丝蚓提 供有利的生长环境. 此外, 姚思鹏等 ${ }^{[9]}$ 和 $\mathrm{Yu}$ 等 ${ }^{[10]}$ 的研究认为水丝蚓可直接利用微囊藻碎屑作为食物. 本试 验系统内水丝蚓孵化于太湖梅梁湾的沉积物, 而梅梁湾是太湖富营养化最严重的区域之一, 频繁暴发蓝藻 水华, 由于水丝蚓对环境的长期适应, 早已形成了稳定的摄食习性, 并且姚思鹏等 ${ }^{[9]}$ 的同位素试验结果表 明, 在栅藻和微囊藻同时存在的情况下, 水丝蚓仅摄食微囊藻, 说明水丝蚓对微囊藻碎屑具有一定的摄食偏 向. 这就不难解释本试验组微囊藻碎屑加人后, 水丝蚓的生物量逐渐增加. 而水丝蚓生物量呈先升后降的变 化趋势, 与水丝蚓的生活史特征有关. Kennedy ${ }^{[27]}$ 发现霍甫水丝蚓 (Limnodrilus hoffmeister) 的繁殖周期为 $2 \sim$ 3 周, 而且大多数个体在繁殖后死亡, 只有少数个体存活并再次繁殖. 因此, 在本研究中, 第 28 天的水丝蚓生 物量下降可能与其繁殖后出现大量个体死亡有关.

微囊藻水华暴发后期, 大量的微囊藻碎屑腐烂分解, 最直接的影响是增加水体的营养盐负荷, 微囊藻碎 屑能向水体释放大量的营养盐 ${ }^{[11]}$. 本研究结果同样发现, 在微囊藻碎屑加人系统后, 水体氮、磷浓度迅速升 高 (图 2 , 图 3), 其中主要以溶解态形式存在, 这将成为水生生物的重要营养来源 ${ }^{[9]}$, 直接导致本研究中浮游 植物生物量的急剧上升. 另外, TN 和 TDN 浓度持续较高, 试验期间始终显著高于初始浓度, 并且较磷营养盐 的持续时间长. 而磷营养盐在试验期间, 呈现先升后降最后继续升高的趋势, 与磷的地球化学性质分不开. 微囊藻碎屑分解释放大量的磷, 使得磷营养盐在试验第 1 天显著升高, 之后由于磷营养盐的沉降作用, 出现 下降的趋势, 在试验第 14 天, 磷营养盐再一次达到峰值, 由于浮游植物和附着藻类在第 14 天生物量呈下降 趋势, 减少了对系统内磷的吸收; 另外消亡的浮游植物和附着藻细胞腐烂分解, 将释放营养盐, 引起水体磷 浓度的再次上升. 微囊藻碎屑的加人, 使得系统内氮、磷营养盐浓度显著上升. 因此, 在微囊藻碎屑分解的环 境下, 条件合适时, 有引发微囊藻水华再次暴发的风险. 本研究也发现在微囊藻碎屑加人系统以后, 在试验 开始的第 9 天, 平均水体 Chl. $a$ 浓度高达 $56 \mu \mathrm{g} / \mathrm{L}$, 最高值为 $105 \mu \mathrm{g} / \mathrm{L}$, 肉眼可见系统表面形成一层薄薄的藻 类水华. 
营养盐是影响浮游植物生长的关键因子 ${ }^{[28]}$, 微囊藻碎屑加人后, 短期内释放了大量的溶解态营养盐, 以 $\mathrm{NH}_{3}-\mathrm{N}$ 为主, 其作为浮游植物最容易利用的氮源 ${ }^{[29]}$, 将很快被水体中浮游藻类吸收利用. 在本试验第 $5 \sim 9$ 天, 随着氮、磷营养盐的增加, 系统内 Chl. $a$ 平均浓度呈上升趋势. 但在整个试验过程中, Chl. $a$ 的平均浓度未 发生显著变化, 系统内的双壳类底栖动物通过滤食水体中的浮游藻类 ${ }^{[30-32]}$, 从而抑制了浮游植物的生长, 使 本试验系统内的浮游植物生物量保持在一个相对稳定的水平.

附着藻作为水体重要的初级生产者之一, 其生长与水体营养盐和光照强度密不可分. 研究表明, 附着藻 生物量随水体氮浓度的升高而增加 ${ }^{[1,33]}$. 本试验在加人微囊藻碎屑后, 水体氮营养盐浓度不断增加, 而我们 发现附着藻生物量却呈逐渐下降的趋势. 本试验中附着藻生物量的下降, 可能受水下光照强度变化的影响. 微囊藻碎屑加人后, 浮游植物生物量上升, 削弱了水下光照强度, 从而抑制了附着藻类的生长. 另外, 椭圆萝 卜螺和铜锈环棱螺也可刮食桶壁附着藻 ${ }^{[34-35]}$, 因此附着藻的生长繁殖还受到捕食者威胁. 但在试验开始前, 系统经过一个月的稳定沉降, 试验桶内各生物初始生物量较为稳定, 而附着藻生物量的显著下降发生在微 囊藻碎屑添加人系统之后, 且观察发现试验系统水色呈绿色, 浮游植物 Chl. $a$ 浓度也与此一致. 因此, 我们认 为浮游植物的遮光作用, 是本试验附着藻生物量下降的主要原因.

在近几十年里, 太湖频繁的暴发大面积的蓝藻水华 ${ }^{[36]}$, 且水华的持续时间也越来越长 ${ }^{[37]}$, 我们的研究 结果将对湖泊管理和蓝藻水华管控提供重要的理论依据. 本研究中, 微囊藻碎屑不仅通过分解产生大量营 养盐刺激初级生产力升高 (浮游植物 Chl. $a$ ) ; 而且还可促进次级生产力 (水丝蚛) 的增长. 微囊藻是太湖常 见的蓝藻水华种, 而之前的研究发现微囊藻碎屑 $70 \%$ 的氮源进人了太湖底栖食物网 ${ }^{[38]}$. 而在太湖, 风浪对 沉积物的扰动作用很强, 这不仅加速了沉积物营养盐释放速率 ${ }^{[39]}$, 而且还可将未分解完全的蓝藻碎屑再次 带人水中, 在微生物等的作用下, 将进一步促进蓝藻碎屑营养盐的释放. 这些蓝藻碎屑分解产生的营养盐, 主要以溶解态形式存在, 则很容易被蓝藻利用而再次形成蓝藻水华. 蓝藻碎屑通过促进底栖次级生产力增 长, 不仅影响底栖食物网结构, 而且还可能加速了沉积物营养盐向水体释放的速率, 因为底栖动物 (包括水 丝蚓) 通过扰动沉积物可显著增加水体营养盐浓度 ${ }^{[40-43]}$, 这可能反过来加速蓝藻水华的暴发速度 (加速营养 盐供给).

\section{4 结论}

1) 微囊藻碎屑可显著增加水体不同形态氮、磷营养盐浓度, 其中高浓度氮的持续时间较磷长, 且主要以 溶解态氮的形式存在.

2 ) 浮游植物生物量 (Chl. $a$ 浓度) 随营养盐浓度的升高而上升, 试验期间在水体表面出现大量藻类聚集 的现象.

3) 微囊藻碎屑的加人, 不仅引起较高的浮游初级生产力 (Chl. $a$ 浓度), 而且还引起底栖次级生产力 (水 丝蚓生物量) 的上升. 我们的研究结果证明了微囊藻碎屑的分解对水质及部分水生生物生物的影响: 微囊 藻分解不仅直接影响水质, 增加水体氮磷营养盐, 还能直接或间接影响浮游植物、附着藻和底栖生物的生 物量.

致谢：感谢李柯、周德勇、刘旭博、姚思鹏、章铭、姬娅婵对受控试验和实验室工作的支持.

\section{5 参考文献}

[ 1 ] Qin BQ, Yang LY, Chen FZ et al. Lake eutrophication mechanism, control technology and its application. Chinese Science Bulletin, 2006, 51(16)：1857-1866. [ 秦伯强, 杨柳燕, 陈非洲等. 湖泊富营养化发生机制与控制技术及其应用. 科学通报, 2006, 51(16): 1857-1866.]

[ 2 ] Kong FX, Ma RH, Gao JF et al. The theory and practice of prevention, forecast and warning on cyanobacteria bloom in Lake Taihu. J Lake Sci, 2009, 21 (3) : 314-328. DOI: 10.18307/2009.0302. [孔繁翔, 马荣华, 高俊峰等. 太湖蓝藻 水华的预防、预测和预警的理论与实践. 湖泊科学, 2009, 21(3) : 314-328.]

[ 3 ] Zhu GW. Eutrophic status and causing factors for a large, shallow and subtropical Lake Taihu, China. J Lake Sci, 2008, 20(1) : 21-26. DOI: 10.18307/2008.0103. [ 朱广伟. 太湖富营养化现状及原因分析. 湖泊科学, 2008, 20 (1): 21-26.] 
[ 4 ] Hamill KD. Toxicity in a benthic freshwater cyano-bacteria (blue-green algae) : first observations in New Zealand. New Zealand Journal of Marine and Freshwater Research, 35: 1057-1069. DOI: 10.1080/00288330.2001.9517062.

[ 5 ] Dawson RM. The toxicology of microcystins. Toxicon, 1998, 36: 953-962. DOI: 10.1016/s0041-0101(97)00102-5.

[ 6 ] Li DH, Li BG, Wang GH et al. Effect of water bloom-forming cyanobacterial bio-substances on the growth of submerged macrophyte Ceratophyllum oryzetorum Kom. Acta Hydrobiologica Sinica, 2007, 31(5): 689-692. [李敦海, 李根保, 王 高鸿等. 水华蓝藻生物质对沉水植物五刺金鱼藻生长的影响. 水生生物学报, 2007, 31(5): 689-692.]

[ 7 ] Kong FX, Gao G. Hypothesis on cyanobacteria bloom-forming mechanism in large shallow eutrophic lakes. Acta Ecologica Sinica, 2005, 25(3) : 589-595. [ 孔繁翔, 高光. 大型浅水富营养化湖泊中蓝藻水华形成机理的思考. 生态学报, $2005,25(3): 589-595$.]

[ 8 ] Chen YW, Fan CX, Teubner K et al. Changes of nutrients and phytoplankton chlorophyll-a in a large shallow lake, Taihu, China. an 8-year investigation. Hydrobiologia, 2003, 506-509(1/2/3).

[ 9 ] Yao SP. The feeding of Limnodrilus hoffmeisteri and its impact on nutrient cycling between water-sediment [Dissertation]. Wuhan: Huazhong Agricultural University, 2011. [姚思鹏. 霍甫水丝蚓的摄食及其对水一沉积物界面营养盐循环的 影响[学位论文]. 武汉: 华中农业大学, 2011.]

[10] Yu JL, Li YM, Liu XL et al. The fate of cyanobacterial detritus in the food web of Lake Taihu. a mesocosm study using ${ }^{13} \mathrm{C}$ and ${ }^{15} \mathrm{~N}$ labeling. Hydrobiologia, 2013, 710(1) : 39-46. DOI: 10.1007/s10750-012-1205-y.

[11] Li K, Guan BH, Liu ZW. Experiments on decomposition rate and release forms of nitrogen and phosphorus from the decomposing cyanobacterial detritus. J Lake Sci, 2011, 23(6) : 919-925. DOI: 10.18307/2011.0614. [李柯, 关保华, 刘 正文. 蓝藻碎屑分解速率及氮磷释放形态的实验分析. 湖泊科学, 2011, 23(6) : 919-925.]

[12] Sun YJ. Study on the aerobic decomposition and nutrient release of cyanobacteria detritus in Dianshan Lake. China Environmental Science, 2013, 33(11): 2047-2052. [孙远军. 淀山湖蓝藻碎屑的好氧降解和营养盐释放规律研究. 中国 环境科学, 2013, 33(11): 2047-2052.]

[13] Xu H, Cai YJ, Tang XM et al. Community structure of macrozoobenthos and the evaluation of water environment in Lake Taihu. J Lake Sci, 2015, 27 (5) : 840-852. DOI: 10.18307/2015.0510. [许浩, 蔡永久, 汤祥明等. 太湖大型底栖动 物群落结构与水环境生物评价. 湖泊科学, 2015, 27(5): 840-852.]

[14] Zhang Y, Liu L, Cai YJ et al. Benthic macroinvertebrate community structure in rivers and streams of Lake Taihu Basin and environmental determinants. China Environmental Science, 2015, 35(5): 1535-1546. [张又, 刘凌, 蔡永久等. 太 湖流域河流及溪流大型底栖动物群落结构及影响因素. 中国环境科学, 2015, 35(5): 1535-1546.]

[15] Sun X. The influence of zoobenthos on community structure and function of nitrogen transformation microbe in sediment from eutrophic lakes [Dissertation]. Nanjing: Nanjing University, 2015. [孙旭. 富营养化湖泊底栖动物对沉积物氮转 化菌群落结构和功能的影响 [学位论文]. 南京: 南京大学, 2015.]

[16] Xie Z. Macroinvertebrate communitie structure and their responses to environmental factors in Taihu Lake, China [Dissertation]. Nanjing: Nanjing Agricultural University, 2014. [谢钊. 太湖大型底栖无脊椎动物群落结构特征及其对环境 因子的响应 [学位论文]. 南京: 南京农业大学, 2014.]

[17] Sun X, He XY, Yang LY. The effect of algal bloom on the emission flux of nitrous oxide from Chironomus plumosus larvae. J Lake Sci, 2018, 30(4) : 1019-1026. DOI: 10.18307/2018.0415. [孙旭, 何晓云, 杨柳燕, 蓝藻水华对羽摇蚊 (Chironomus plumosus) 幼虫 $\mathrm{N}_{2} \mathrm{O}$ 排放通量的影响. 湖泊科学, 2018, 30(4) : 1019-1026.]

[18] Zhang LY, Li KY, Liu ZW et al. Sedimented cyanobacterial detritus as a source of nutrient for submerged macrophtes (Vallisneria spiralis and Elodea nuttallii): An isotope experiment using ${ }^{15}$ N. Limnology and Oceanography, 2010, 55 (5) : 1912-1917. DOI: 10.4319/lo.2010.55.5.1912.

[19] Cai YJ, Gon ZJ, Qin BQ. Community structure and diversitv of macrozoobenthos in Lake Taihu, a large shallow eutrophic lake in China. Biodiversity Science, 2010, 18(1) : 50-59. DOI: 10.3724/SP.J.1003.2010.050. [蔡永久, 龚志军, 秦伯 强. 太湖大型底栖动物群落结构及多样性. 生物多样性, 2010, 18(1) : 50-59.]

[20] Cai YJ, Jian JH, Zhang L et al. Community structure and biodiversity of macrozoobenthos of typical lakes in the middle and lower reaches of the Yangtze River. J Lake Sci, 2010, 22(6) : 811-819. DOI: 10.18307/2010.0602. [蔡永久, 姜加 虎, 张路等. 长江中下游湖泊大型底栖动物群落结构及多样性. 湖泊科学, 2010, 22(6) : 811-819.]

[21] Cai W, Cai YJ, Gong ZJ et al. Temporal and spatial patterns of Corbicula fluminea in Lake Taihu. J Lake Sci, 2010, 22 (5) : 714-722. DOI: 10.18307/2010.0513. [ 蔡炜, 蔡永久, 龚志军等. 太湖河蚬时空格局. 湖泊科学, 2010, 22 
(5): 714-722.]

[22] Qin BQ, Zhu GW, Gao G et al. A drinking water crisis in Lake Taihu, China: linkage to climatic variability and lake management. Environmental Management, 2010, 45: 105-112. DOI: 10.1007/s00267-009-9393-6.

[23] Jin XC, Tu QY eds. The standard methods in lake eutrophication investigation. Beijing: China Environmental Science Press, 1990. [ 金相灿, 屠清瑛. 湖泊富营养化调查规范. 北京: 中国环境科学出版社, 1990. ]

[24] Guo WJ, Fu ZY, Wang H et al. The quantitative relation of aquatic parameters and phytoplankton biomass in the process of algal blooms-The case of Meiliang Bay in Taihu Lake. China Environmental Science, 2018, 38(4) : 1517-1525. [郭文 景, 符志友, 汪浩等. 水华过程水质参数与浮游植物定量关系的研究一以太湖梅梁湾为例. 中国环境科学, 2018, 38(4) : 1517-1525.]

[25] Gong ZJ, Xie P, Tang HJ et al. The influence of eutrophycation upon community structure and biodiversity of macrozoobenthos. Acta Hydrobiologica Sinica, 2001, (3) : 210-216. [龚志军, 谢平, 唐汇涓等. 水体富营养化对大型底栖动物 群落结构及多样性的影响. 水生生物学报, 2001, (3) : 210-216.]

[26] Li Y, Cai YJ, Qin BQ et al. Temporal and spatial patterns of Limnodrilus hoffmeisteri Claparède in Lake Taihu. J Lake Sci, 2012, 24(3) : 450-459. DOI: 10.18307/2012.0318. [李艳, 蔡永久, 秦伯强等. 太湖霍甫水丝蚓 (Limnodrilus hoffmeisteri Claparède) 的时空格局. 湖泊科学, 2012, 24(3): 450-459.]

[27] Kennedy CR. The life history of Limnodrilus hoffmeisteri Clap. (Oligochaeta. Tubificidae) and its adaptive significance. Oikos, 1966, 17: 158-168. DOI: 10.2307/3564940.

[28] Chapman BR, Ferry BW, Ford TW. Phytoplankton communities in water bodies at Dungeness, U. K. analysis of seasonal changes in response to environmental factors. Hydrobiologia, 1997, 362(1-3). DOI: 10.1023/ A: 1003190702786.

[29] Yang L, Zhang M, Liu ZW. Uptake of various forms of nitrogen by phytoplankton community in spring in Lake Taihu. $J$ Lake Sci, 2011, 23(4) : 605-611. DOI: 10.18307/2011.0417. [杨柳, 章铭, 刘正文. 太湖春季浮游植物群落对不同 形态氮的吸收. 湖泊科学, 2011, 23(4): 605-611.]

[30] Jeppesen E, Søndergaard M, Lauridsen TL et al. Biomanipulation as a restoration tool to combat eutrophication: recent advances and future challenges. Advances in Ecological Research, 2012, 47: 297-315. DOI: 10.1016/B978-0-12-398315-2. 00006-5.

[31] Yang DM, Chen YW, Liu ZW et al. Top-down effects of Anodonta woodiana on nutrient concentration \& phytoplankton community composition in a microcosm ecosystem. J Lake Sci, 2008, 20(2) : 228-234. DOI: 10.18307/2008.0215. [杨 东妹, 陈宇炜, 刘正文等. 背角无齿蚌滤食对营养盐和浮游藻类结构影响的模拟. 湖泊科学, 2008, 20(2): 228-234.]

[32] Reeders HH, Vaate AB. Zebra mussels (Dreissena polymorpha) : a new perspective for water quality management. Hydrobiologia, 1990, 200-201 : 437-450. DOI: 10.1007/BF02530361.

[33] Tan BC, Cai YJ, An M et al. Effects of nitrogen enrichment on the growth and elemental stoichiometry of periphyton. Ecology and Environmental Sciences, 2016, 25(8): 1376-1381. [谈冰畅, 蔡永久, 安苗等. 水体氮质量浓度升高对附着 藻生长和元素计量特征的影响. 生态环境学报, 2016, 25(8): 1376-1381.]

[34] Tan BC, Li KY, An M et al. Effects of increasing nitrogen loading on the interactions among Bellamya aeruginosa, periphytic algae and Vallisneria spiralis. Acta Scientiae Circumstantiae, 2015, 35(11) : 3704-3709. DOI: 10.13671/j.hjkxxb. 2015.0069. [谈冰畅, 李宽意, 安苗等. 氮负荷升高对螺-附着藻-苦草生态关系的影响. 环境科学学报, 2015, 35 (11) : 3704-3709.]

[35] Li KY, Liu ZW, Li CH et al. Food sources of snail Radix swinhoei in Lake Taihu. J Lake Sci, 2008, 20(3) : $339-343$. DOI : 10.18307/2008.0312. [李宽意, 刘正文, 李传红等. 太湖椭圆夢卜螺的食物来源分析. 湖泊科学, 2008, 20 (3) : 339-343.]

[36] Duan HT, Ma RH, Xu XF et al. Two-decade reconstruction of algal blooms in China's Lake Taihu. Environmental Science \& Technology, 2009, 43(10). DOI: 10.1021/es8031852.

[37] Ma JR, Qin BQ, Paer HW et al. The persistence of cyanobacterial (Microcystis spp. ) blooms throughout winter in Lake Taihu, China. Limnology and Oceanography, 2016, 61(2). DOI: 10.1002/lno.10246.

[38] Yu JL, He H, Liu ZW et al. Cyanobacteria-derived nitrogen uptake by benthic invertebrates in Lake Taihu. a mesocosm study using ${ }^{15} \mathrm{~N}$ labeling. Knowledge and Management of Aquatic Ecosystems, 2014, 415 ( 7 ). DOI: 10. 1051/ $\mathrm{kmae} / 2014033$. 
[39] Qin BQ, Hu WP, Gao G et al. Dynamics of sediment resuspension and the conceptual schema of nutrient release in the large shallow Lake Taihu, China. Chinese Science Bulletin, 2004, 49(1). DOI: 10.1007/bf02901743.

[40] Zhang L, Gu XZ, Shao SG et al. Impacts of Asian Clams (Corbicula fluminea) on lake sediment properties and phosphorus movement. Environmental Science, 2011, 32(1) : 88-95. [张雷, 古小治, 邵世光等. 河蚬 (Corbicula fluminea) 扰动 对湖泊沉积物性质及磷迁移的影响. 环境科学, 2011, 32(1): 88-95.]

[41] Jin H, Luo XG, Gu J et al. Inhibiting effect of Corbicula fluminea on the bioturbation of Limnodrilus hoffmeisteri. J Lake $S c i, 2016,28$ (6) : 1348-1353. DOI: 10.18307/2016.06.20. [ 靳辉, 罗旭光, 谷娇等. 河蚬 (Corbicula fluminea) 对霍 甫水丝蚓 (Limnodrilus hoffmeisteri) 生物扰动的抑制效应. 湖泊科学, 2016, 28(6): 1348-1353.]

[42] Zhang L, Gu XZ, Wang ZD et al. The influence of Tubificid worms bioturbation on the exchange of phosphorus across sediment- water interface in lakes. J Lake Sci, 2010, 22(5) : 666-674. DOI: 10.18307/2010.0507. [张雷, 古小治, 王兆德 等. 水丝蚓 (Tubificid worms) 扰动对磷在湖泊沉积物一水界面迁移的影响. 湖泊科学, 2010, 22(5) : 666-674.]

[43] Yao SP, Li K, Zhou DY et al. Impact of Limnodrilus hoffmeristeri on inorganic nitrogen and phosphorus exchange at the sediment-water interface in Meiliang Bay, Lake Taihu. Environmental Science \& Technology, 2011, 34(1) : 100-104. [姚 思鹏, 李柯, 周德勇等. 霍甫水丝蚓对太湖梅梁湾沉积物影响一一水界面无机氮、磷交换. 环境科学与技术, 2011, 34 (1) : 100-104. ] 